Author Manuscript (as accepted by Environmental Science: Processes and Impacts)

\title{
Surface wipe and bulk sampling of household dust: arsenic exposure in Cornwall, UK
}

D. R. S. Middleton, ${ }^{a, b, c}$ M. J. Watts, ${ }^{b}$ E. M. Hamilton, ${ }^{b}$ J. D. Coe, ${ }^{b}$ T. Fletcher ${ }^{c}$ H. Crabbec, R. Close, ${ }^{c}$ G.S. Leonardi ${ }^{\mathrm{c}}$ and D. A. Polya ${ }^{\mathrm{a}}$

${ }^{a}$ School of Earth and Environmental Sciences \& Williamson Research Centre for Molecular Environmental Science, University of Manchester, Manchester, UK, M13 9PL

${ }^{b}$ Inorganic Geochemistry, Centre for Environmental Geochemistry, British Geological Survey, Nottingham, UK, NG12 5GG

${ }^{c}$ Centre for Radiation, Chemicals and Environmental Hazards (CRCE), Public Health England, Chilton, Didcot, Oxfordshire, UK, OX11 ORQ

Correspondence: Michael J. Watts, mwatts@bgs.ac.uk

\begin{abstract}
Dust elemental levels can be expressed as concentrations (bulk samples) or surface loadings (wipe samples). Wipe sampling has not been widely adopted for elements other than lead $(\mathrm{Pb})$. In this study, 433 wipe samples from 130 households in south west England- a region of widespread, natural and anthropogenic arsenic contamination linked with previous mining activities- were analysed to (i) quantify loadings of arsenic (As); (ii) assess the quality of wipe data using QA/QC criteria; (iii) estimate, using published ingestion rates, human exposure to As in dust using loadings and concentrations from 97 bulk samples and (iv) comparatively assess the performance of wipe and bulk sampling using associations with As biomonitoring data (urine, toenails and hair). Good QC performance was observed for wipes: strong agreement between field duplicates, non-detectable contamination of field blank wipes and good reference material recoveries. Arsenic loadings exceeded an existing urban background benchmark in 67 (52\%) households. No exceedances of tolerable daily As intake was observed for adult exposure estimates but infant estimates exceeded for 1 household. Infant estimates calculated using bulk concentrations resulted in 4 (3\%) exceedances. Neither wipe nor bulk As metrics were sufficiently better predictors of As in biospecimens. Sampling strategies, analytical protocols, exposure metrics and assessment criteria require refinement to validate dust sampling methodologies.
\end{abstract}

This is an author formatted manuscript in its accepted form. Tables can be found at the end of the document after the references. 


\section{Introduction}

Chronic exposure to elevated concentrations of arsenic (As) is detrimental to human health. Several types of cancer (e.g. lung, bladder and skin) are attributable to As exposure ${ }^{1}$, as well as many noncancerous health effects, including diabetes mellitus ${ }^{2}$; cardiovascular disease ${ }^{3}$ and hypertension ${ }^{4}$. Arsenic is categorised by the International Agency for Research on Cancer (IARC) as Group 1 ("carcinogenic to humans") $)^{5}$ and was ranked 1st on the Agency for Toxic Substances and Disease Registry (ATSDR) priority list of hazardous substances in $2015^{6}$.

Human exposure to As varies by source and pathway. The most widespread route of exposure for As is from the ingestion of contaminated drinking water ${ }^{1}$. Exposure can also occur via the ingestion of contaminated foodstuffs and the ingestion/inhalation of soil and dust, although the relative importance of the latter in non-occupationally exposed settings is a matter for debate. Indoor dust contaminated as a result of mining and smelting operations is the route of human exposure to As that forms the focus of the present paper. To enable informative exposure assessments of this and other potentially toxic elements, robust, standardised and logistically feasible sampling procedures are needed.

Estimation of elemental quantities in indoor dust can be approached in two ways: (1) calculation of bulk concentrations (mass per unit mass, e.g. $\mu \mathrm{g} \mathrm{g}^{-1}$ ), such as by means of composite vacuum sampling techniques or (2) calculation of surface loadings (mass per unit area, e.g. $\mu \mathrm{g} \mathrm{m}^{-2}$ ) by means of wipe sampling. Sampling using wipes is a routine method employed for quantifying lead $(\mathrm{Pb})$ in residential environments and is a recommended regulatory method in the USA. Indoor Pb loadings using the wipe sampling method have been correlated with child blood $\mathrm{Pb}$ concentrations ${ }^{7}$, screening values for loadings have been suggested ${ }^{8}$ and easily dissolvable wet wipes are commercially available and cost effective. Despite the practical and logistical benefits of wipe sampling (rapid, convenient collection of large sample numbers that can be easily digested and analysed by standard analytical techniques) the method has not been widely adopted for other elements such as As. The applicability of wipe sampling for elements other than $\mathrm{Pb}$ has been assessed by McDonald et al. (2011) ${ }^{9}$ and deemed appropriate for determining multi-element loadings in indoor environments. Wipe sampling has been successfully applied to assess surface loadings of As in environments such as children's play $\operatorname{areas}^{10}$ and urban homes ${ }^{11,12}$. Screening values for As surface loadings have also been proposed ${ }^{8}$ (387 $\mu \mathrm{g} \mathrm{m}^{-2}$ ), but the suitability of As loadings to assess human health exposure still needs elucidating. Dust ingestion rates have been derived ${ }^{13}$ and subsequently adapted ${ }^{14}$ in the context of surface loadings. Such estimates may prove useful in assessing human exposures to As and other elements in indoor dust. 
Cornwall, in south west England, has a history of extensive and widespread mining and smelting. The primary ores of interest were copper $(\mathrm{Cu})$ and tin $(\mathrm{Sn})$, but an estimated 250,000 tonnes of As were also extracted ${ }^{15}$. The scale of mining in Cornwall has left a legacy of contamination ${ }^{16}$. Many studies have reported elevated elemental concentrations in Cornwall; including in residential soil ${ }^{17}$, private water supplies ${ }^{18}$ and household dust ${ }^{17}$. Elevated concentrations of As have been reported in human urine $^{19}$, toenails ${ }^{20,21}$ and hair ${ }^{20,22}$. Consideration of potentially vulnerable sub-groups of the population is warranted, including those living in close proximity to former mining sites and small children who come into contact with indoor dust more frequently due to playing habits and hand-to-mouth activity ${ }^{23}$.

While conducting an investigation ${ }^{24}$ on human As exposure from various sources (water, soil, food and dust) in south west England, household dust was collected by surface wipe sampling. This paper aims to use surface wipe sampling to assess potential human exposure to As at locations in south west England via the ingestion of household dust. Emphasis was placed primarily on the surface wipe sampling (loadings) compared to the composite sampling derived from vacuum cleaners (bulk concentrations). Bulk concentrations used for comparison in this study have already been reported ${ }^{17}$. Specific objectives for this study were:

(i) Determine surface loadings of As using multiple wipe samples collected from households across Cornwall.

(ii) Evaluate performance data (detection limits, reproducibility and recoveries of certified reference material concentrations) to assess the limitations of the wipe methodology.

(iii) Estimate potential human exposure to As from household dust using published dust intake rates (in bulk and loading units) and compare the information yielded by wipe and bulk sampling.

(iv) Compare the relative utility of wipe and bulk sampling by assessing the goodness of fit between As concentrations in biological samples (urine, toenails and hair, whose results have been reported previously $^{19,20}$ ) and either bulk As concentrations or As loadings.

\section{Experimental}

\section{Ethical approval}

The wider study ${ }^{24}$ investigating As exposure in Cornwall was ethically approved by the University of Manchester Research Ethics Committee (Ref 13068) and the NHS National Research Ethics Service (NRES) (Ref 13/EE/0234). 


\section{Sampling methodology}

Participant recruitment and sampling strategy. The recruitment and sampling strategy employed for households participating in the Cornwall-wide study of As exposure are detailed elsewhere ${ }^{19}$. In brief, the target population for the exposure study was Cornish residents using a single domestic private drinking water supply (the exposure route of primary interest ${ }^{18,19,25}$ ) in Cornwall. Households were contacted via mail and followed up with a telephone call. Additional exposure sources assessed were residential soil ${ }^{17}$ and household dust with paired biomonitoring samples (urine ${ }^{19}$, hair and toenails ${ }^{20}$ ). For this paper, the authors describe the testing of dust wipe/bulk dust methodologies employed at 127 households in November 2013 to evaluate dust as a possible source of As exposure. On a separate occasion (April 2015), households living in the direct vicinity of the former Devon Great Consols (DGC) As mining conglomerate were contacted by email. Three households responded and were visited in this location. Figure 1 shows the extent of both the Cornwall-wide and DGC-focused sampling.

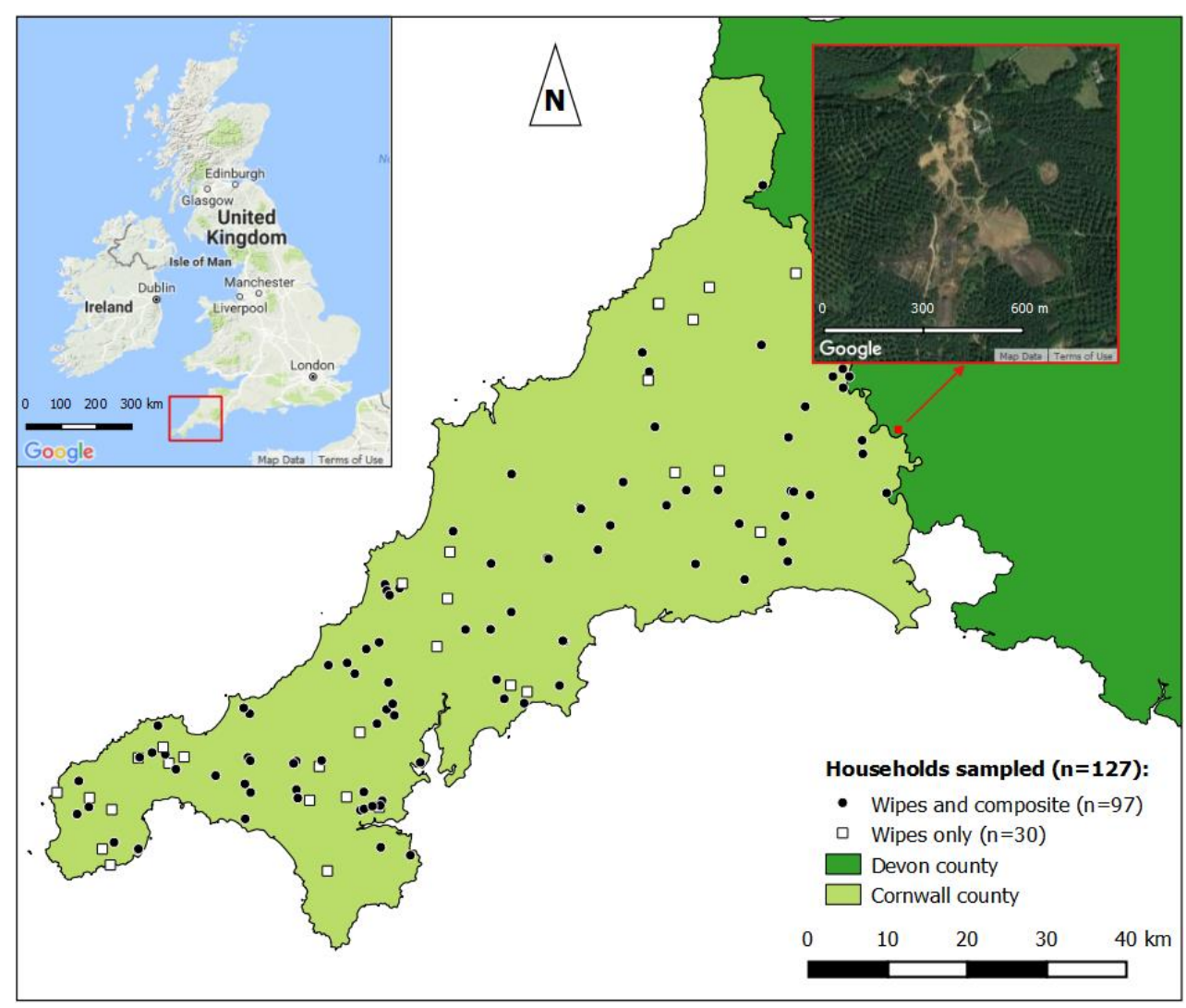

Figure 1 Spatial distribution of sampled households across Cornwall and satellite imagery of the former Devon Great Consols (DGC) mining conglomerate (right inset). To protect participant confidentiality, household points at DGC have not been plotted. Compiled using QGIS 2.18. Contains Ordnance Survey data @ Crown Copyright and database rights 2017. 
Dust wipe and bulk sample collection. Indoor dust wipe samples were collected using Ghost Wipes ${ }^{\mathrm{TM}}$ and a $10 \times 10 \mathrm{~cm}$ plastic template (Environmental Express, Charleston, USA). Participants were asked to clean their household surfaces four weeks prior to sampling visits but not again prior to visit. All wipe samples were collected from raised surfaces (e.g. window sills, shelves, tops of wardrobes). Field teams attempted to collect samples from a range of upstairs and downstairs rooms to obtain a household representative sample. In practice, this was not always possible (see limitations). Wipe samples were collected by the field team using the American Society for Testing Materials (ASTM) E 1728 protocol $^{26}$ and placed into polypropylene DigiTUBETM vessels (SCP Science, Québec, Canada). Powderless nitrile gloves were worn and equal pressure was applied when wiping surfaces in the prescribed S-shaped pattern. Participants were asked to complete a questionnaire to obtain: the date surfaces were cleaned prior to sampling; if there were pets in the house and if any recent renovations to the property had taken place. Bulk household vacuum dust samples were collected by emptying the contents of the household vacuum cleaner into plastic sampling bags. Bulk samples were not collected from households at DGC.

\section{Chemical analysis}

The preparation and analysis of composite vacuum bag dust samples (including analytical QA/QC performance) and that of other samples (As in drinking water, soil, urine, toenail and hair) have been described in detail elsewhere ${ }^{17,19,20}$.

Reagents and standards. Deionised water was of 18.2 M $\Omega$ quality (Millipore, UK). All acids (nitric $\left(\mathrm{HNO}_{3}\right)$, hydrochloric $(\mathrm{HCl})$, hydrofluoric $(\mathrm{HF})$ and perchloric $\left.\left(\mathrm{HClO}_{4}\right)\right)$ and hydrogen peroxide $\left(\mathrm{H}_{2} \mathrm{O}_{2}\right)$ were Romil-SpA ${ }^{\mathrm{TM}}$ grade (Romil, UK). Arsenic calibration standards were from a multielement $1000 \mathrm{mg} \mathrm{L}^{-1}$ PrimAg® grade solution (Romil, UK). Arsenic matrix-matched solutions ( $25 \mu \mathrm{g}$ $\mathrm{L}^{-1}$ ) were prepared from a multi-element solution (Ultra Scientific, USA). A Tellurium (Te) internal standard was prepared from a PlasmaCAL 10,000 $\mathrm{mg} \mathrm{L}^{-1}$ solution (SCP Science, Canada).

Dust wipe dissolution. Wipes were uncapped and loaded into a graphite hot block in the DigiTUBETM $_{\mathrm{S}}$ in which they were collected. Ten millilitres of $50 \% \mathrm{v} / \mathrm{v} \mathrm{HNO}_{3}$ was added to each tube and left to stand for 10 minutes. Four millilitres of concentrated $\mathrm{HNO}_{3}+2.5 \mathrm{~mL}$ of concentrated $\mathrm{HCl}$ $+1 \mathrm{~mL}$ of concentrated HF were added before covering tubes with disposable polypropylene watch glasses (SCP Science, Québec, Canada), ramping the temperature to $70^{\circ} \mathrm{C}$ and leaving overnight. Watch glasses were removed before drying down at $110^{\circ} \mathrm{C}$ and then cooling to $50^{\circ} \mathrm{C}$. Four millilitres of concentrated $\mathrm{HNO}_{3}+1 \mathrm{~mL}$ of $\mathrm{H}_{2} \mathrm{O}_{2}$ were added and the temperature was ramped to $90^{\circ} \mathrm{C}$ to dry solutions down to a gel. Digests were reconstituted by adding $1 \mathrm{~mL}$ of $50 \% \mathrm{v} / \mathrm{v} \mathrm{HNO}_{3}$, heating to $50^{\circ} \mathrm{C}$ for 20 minutes before adding $9 \mathrm{~mL}$ of deionized water. 
Total arsenic determination. Wipe digests were diluted $\times 5$ with $1 \% \mathrm{v} / \mathrm{v} \mathrm{HNO}_{3}+0.5 \% \mathrm{v} / \mathrm{v} \mathrm{HCl}$ and analysed for total As concentrations by inductively coupled plasma mass spectrometry (ICP-MS) (Agilent $7500 \mathrm{cx}$ series) using previously reported instrumental operating conditions ${ }^{27}$. A four-point calibration $\left(0,1,10\right.$ and $\left.100 \mu \mathrm{g} \mathrm{L}^{-1}\right)$ and helium $(\mathrm{He})$ collision cell mode were used, the latter to reduce the potential polyatomic interference of argon chloride $\left({ }^{40} \mathrm{Ar}^{35} \mathrm{Cl}^{+}\right)$on ${ }^{75} \mathrm{As}^{+}$. A multi-element internal standard was introduced via a T-piece. An arsenic signal enhancement was caused by high carbon concentrations in dust wipe matrices. Concentrations of $>15,000 \mathrm{mg} \mathrm{L}^{-1}$ of non-purgeable organic carbon (NPOC) were determined by a Shimadzu TOC CPH instrument in blank wipe digest matrices. To correct for this enhancement, Te was selected as the internal standard as its first ionisation energy is closest to As. Doubly-charged ${ }^{150} \mathrm{Nd}^{++}$and ${ }^{150} \mathrm{Sm}^{++}$interferences on As were corrected using single element standards at $100 \mu \mathrm{g} \mathrm{L}^{-1}$ and the application of a correction factor as described previously ${ }^{27}$.

Quality assurance and control. The ICP-MS auto-sampler was housed in a protective cover to avoid contamination throughout analysis. Quality controls were monitored via the inclusion of field wipe blanks, field duplicate and Certified Reference Material (CRM) -spiked wipe samples and reagent and laboratory wipe blanks. Field wipe blanks were unpackaged, unfolded/refolded and placed into DigiTUBE $^{\mathrm{TM}_{\mathrm{S}}}$ in the same manner as sample wipes without wiping any surfaces. Duplicate wipes were collected by placing the template directly adjacent to the location of the corresponding sample. Digestion batches (12 in total) of 48 tubes consisted of 6 CRM-spiked wipes, 3 reagent blanks, 3 lab wipe blanks and 36 wipes collected in the field. The CRMs used were National Institute of Standards and Technology (NIST) 2584 Indoor Dust and NIST 2711a Montana II Soil. Spiked wipes were unfolded onto clean weighing boats and a specified mass (NIST 2584: $0.1 \mathrm{~g}$; NIST 2711a: $0.05 \mathrm{~g}$ ) of material was placed in the centre before refolding and placing into DigiTUBE ${ }^{\mathrm{TM}_{\mathrm{S}}}$ for digestion ( 3 of each CRM per batch). Reagent blanks consisted of the digestion matrix in empty DigiTUBE ${ }^{\mathrm{TM}_{\mathrm{S}}}$ and

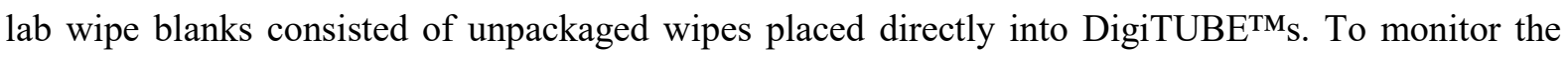
effectiveness of the Te internal standard in correcting for As signal enhancement, matrix-matched As solutions (25 As $\mu \mathrm{g} \mathrm{L}^{-1}$ ) were prepared in a lab wipe blank matrix. Wipe As concentrations were blank sequentially corrected by analytical run blanks, reagent blanks and lab blank wipes. The As loading limit of detection (LOD) was calculated as 3-times the SD of laboratory wipe blanks. Loadings below the LOD were censored with $0.5 \times$ LOD.

\section{Data analysis}

Surface arsenic loading calculations. Total As loadings $\left(\mu \mathrm{g} \mathrm{m}^{-2}\right)$ were calculated as follows:

Loading $=(\mathrm{CDig} \times \mathrm{VDig}) /(\mathrm{A})$, 
where CDig is the As concentration $\left(\mu \mathrm{g} \mathrm{mL} L^{-1}\right)$ of the digest solution, VDig is the final volume $(\mathrm{mL})$ of the digest solution prior to dilution for analysis, A is the area of surface wiped $\left(\mathrm{m}^{2}\right)$.

Statistical analysis and spatial mapping. Data management, the calculation of summary statistics (maximum, minimum and mean As loadings and concentrations) and As intake rates were conducted in Microsoft Excel 2010. Statistical plots, multiple linear regression (natural log-transformed variables), Pearson and Spearman correlation analysis with corresponding significance tests and confidence intervals were generated in $\mathrm{R}$ version $3.3 .1^{28}$ through the RStudio interface ${ }^{29}$. Spatial mapping was conducted using Quantum GIS (QGIS) version 2.18.

Human arsenic exposure estimates. Household mean As loadings and composite vacuum concentrations were used to calculate daily As intake estimates from dust using recently published dust intake estimates (both in units of surface area per day ${ }^{13}$ and units of mass per day ${ }^{14}$ ). Daily intakes were calculated separately for two exposure extremes: infants (0-6 months) and adults (20-59 years) and discussed in the context of the FAO/WHO JEFCA tolerable daily intake ${ }^{1}$ of $3.0 \mathrm{As} \mu \mathrm{g} \mathrm{kg}^{-1}$ (BW) day $^{-1}$.

Arsenic intake rates were calculated as follows:

In the context of surface loadings:

$\mathrm{IR}_{\mathrm{As}}=\left(\mathrm{L}_{\mathrm{As}} \times \mathrm{IR}_{\mathrm{d}}\right) / \mathrm{BW}$,

where $\mathrm{IR}_{\mathrm{As}}$ is the As intake rate $\left(\mu \mathrm{g} \mathrm{kg}^{-1}(\mathrm{BW}) \mathrm{day}^{-1}\right), \mathrm{L}_{\mathrm{As}}$ is the As loading $(\mu \mathrm{g} \mathrm{m}-2)$ measured using wipe sampling, $I_{d}$ is the dust ingestion rate, in surface area units $\left(\mathrm{m}^{2}\right.$ day $\left.^{-1}\right)$, corresponding to the age groups for which they were derived and BW is the bodyweight $(\mathrm{kg})$ corresponding to the same age groups. Nominal default bodyweights were consistent with those used in the UK Contaminated Land Exposure Assessment (CLEA) model $^{30}$ and were 70 and $5.6 \mathrm{~kg}$ for adults and infants, respectively.

In the context of bulk concentrations:

$\mathrm{IR}_{\mathrm{As}}=\left(\mathrm{C}_{\mathrm{As}} \times \mathrm{IR}_{\mathrm{b}}\right) / \mathrm{BW}$

where, $C_{A s}$ is the bulk As concentration ( $\mu \mathrm{g} \mathrm{g}^{-1}$ ) measured in composite vacuum samples and $\mathrm{IR}_{\mathrm{b}}$ is the dust ingestion rate in bulk concentration units $\left(\mathrm{g} \mathrm{day}^{-1}\right)$. The previously derived ${ }^{13,14}$ dust ingestion rates that were adopted for this study are shown in Table 1.

Dust ingestion rates were, to our knowledge, the only available in both mass and loading units that were derived using the same approach. No dust ingestion estimates were available for specific sampling locations, e.g. ingestion from raised surfaces relative to floors. 


\section{Results and discussion}

\section{Method performance and practical considerations}

Three hundred and fifty one individual dust wipes were collected from 127 households across Cornwall, plus 28 (7\%) field blanks and 30 (7\%) duplicates, exceeding the recommended 5\% in the ASTM E 1728 protocol $^{26}$ - a total of 409 samples. The mean number of wipes (excluding blanks and duplicates) collected per household was 3 and ranged from 1 to 7 . From the three households visited separately at DGC, 24 individual wipes were collected, 3 (13\%) of which were field blanks and 6 (25\%) of which were duplicates - yielding 15 sample wipes and a mean of 5 per household (range: 36). Cornwall-wide wipes were digested and analysed on a separate occasion to those from DGC. Duplicate agreements and CRM recoveries did not differ between analytical sets, thus pooled results are reported. Lab wipe blanks used to correct data yielded different LODs between the two sample sets and are reported separately.

Limits of detection and field blank performance. The LODs calculated for Cornwall-wide and DGC dust wipes were 2 and 19 As $\mu \mathrm{g} \mathrm{m}^{-2}$, respectively. These differences in detection capability between analysis batches, and in comparison to the much lower LOD reported ${ }^{9}$ by McDonald et al. (0.15 As $\mu \mathrm{g} \mathrm{m}^{-2}$ ) highlight the susceptibility of lab wipe blanks to contamination; either due to handling or already present. Background contamination of the Ghost Wipe ${ }^{\mathrm{TM}}$ brand used in this study has been reported in the literature ${ }^{31}$. Of the 31 field wipe blanks collected from both sites, As loadings were $<$ LOD, with the exception of one wipe blank in which the loading was marginally >LOD. The sampling protocols employed were deemed sufficient in minimising contamination of wipe samples collected in the field.

Precision. Seven out of the eight times that a sample or its duplicate was $<$ LOD, so too was its paired counterpart. Therefore, to prevent the artificial improvement of data censoring on duplicate analyses, only duplicate pairs in which both wipe loadings were >LOD were included. Figure 2 shows the field duplicate agreement of As loadings, expressed as Spearman's correlation coefficient $(\rho)$ : $\rho=0.94$; $\mathrm{n}=28$ loadings (including censored pairs gave a correlation of 0.97 ). Correlations were strong and significant to $\mathrm{P}<0.001$. These duplicate wipe correlations are comparable to those previously reported ${ }^{9}$ for As $(\rho=0.83)$. 


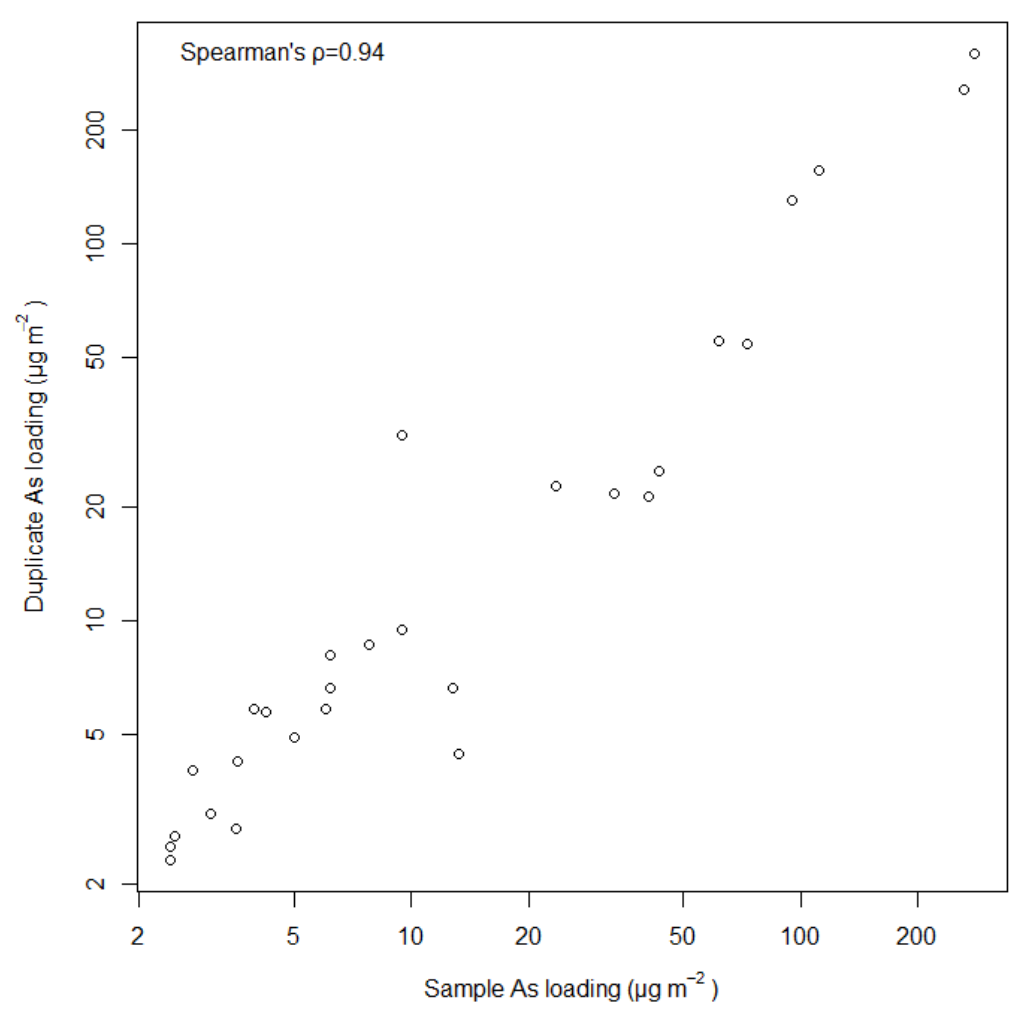

Figure 2 Agreement between As loadings in field duplicate wipes.

Extraction efficiency. Mean percentage recoveries of As from CRM-spiked dust wipes (NIST 2584 and NIST 2711a) and matrix-matched As solutions are presented in Table 2.

Fit-for-purpose As recoveries were achieved for both NIST $2584(86 \pm 13 \%)$ and NIST 2711a $(107 \pm 8 \%)$ CRMs. Arsenic recoveries from matrix-matched As solutions (see experimental) were poorer $(81 \pm 3 \%)$. The Te standardisation applied to As may have over-corrected concentrations, but recoveries were much improved post-correction. Alternatively, the blank correction applied to As concentrations may have accounted for the $5 \mu \mathrm{g} \mathrm{L}^{-1}$ discrepancy between spike recoveries and target concentrations if wipes used in the preparation of matrix matched As solutions did not contain the 5 $\mu \mathrm{g} \mathrm{L}^{-1}$ As background. McDonald et al. did not report such high background concentrations of As in blank wipes ${ }^{9}$, hence their lower reported LOD for As $\left(0.15 \mu \mathrm{g} \mathrm{m}^{-2}\right)$.

\section{Total arsenic loadings}

The degree of within-household variation in wipe loadings was high. Wipe loadings varied by a maximum of 2,953 As $\mu \mathrm{g} \mathrm{m}^{-2}$ within the same household. The mean within-household loading range was $63 \mathrm{As} \mu \mathrm{g} \mathrm{m}^{-2}$, demonstrating that sampling locations highly influenced individual wipe loadings. 
Summary statistics for the individual wipe sample As loadings are presented in Table 3 and the histogram in Figure 3. An upper-benchmark loading (also shown in Figure 3) was previously derived ${ }^{9}$ for As (and other elements) in a study of Canadian urban homes. These were defined statistically (the upper breakpoint of Q-Q normality plots) as values above which loadings were elevated relative to the background. In the present study, in Cornwall, the proportion of households above this Canadian benchmark loading (25 As $\mu \mathrm{g} \mathrm{m}^{-2}$ ) was 58 wipes from 33 (26\%) households. This finding demonstrates that elemental loadings in household dust from rural locations across Cornwall are elevated relative to an existing urban background. At DGC, a higher proportion of upper-benchmark exceedance was observed: 9 wipes from three households. These three households are in close proximity to large amounts of uncovered mine tailings. The household situated closer $(<100 \mathrm{~m})$ to tailings dumps at DGC had considerably higher mean household loadings of As (246 $\mu \mathrm{g} \mathrm{m}^{-2}$ ) compared to two households (39 and $21 \mathrm{As} \mu \mathrm{g} \mathrm{m}^{-2}$ ) that were $400 \mathrm{~m}$ from mine tailings. While insufficient households were sampled at DGC to enable statistical investigation of such an association in the present study, an inverse correlation between soil As concentrations and distance from former mining sites was previously reported in Cornwall ${ }^{17}$.

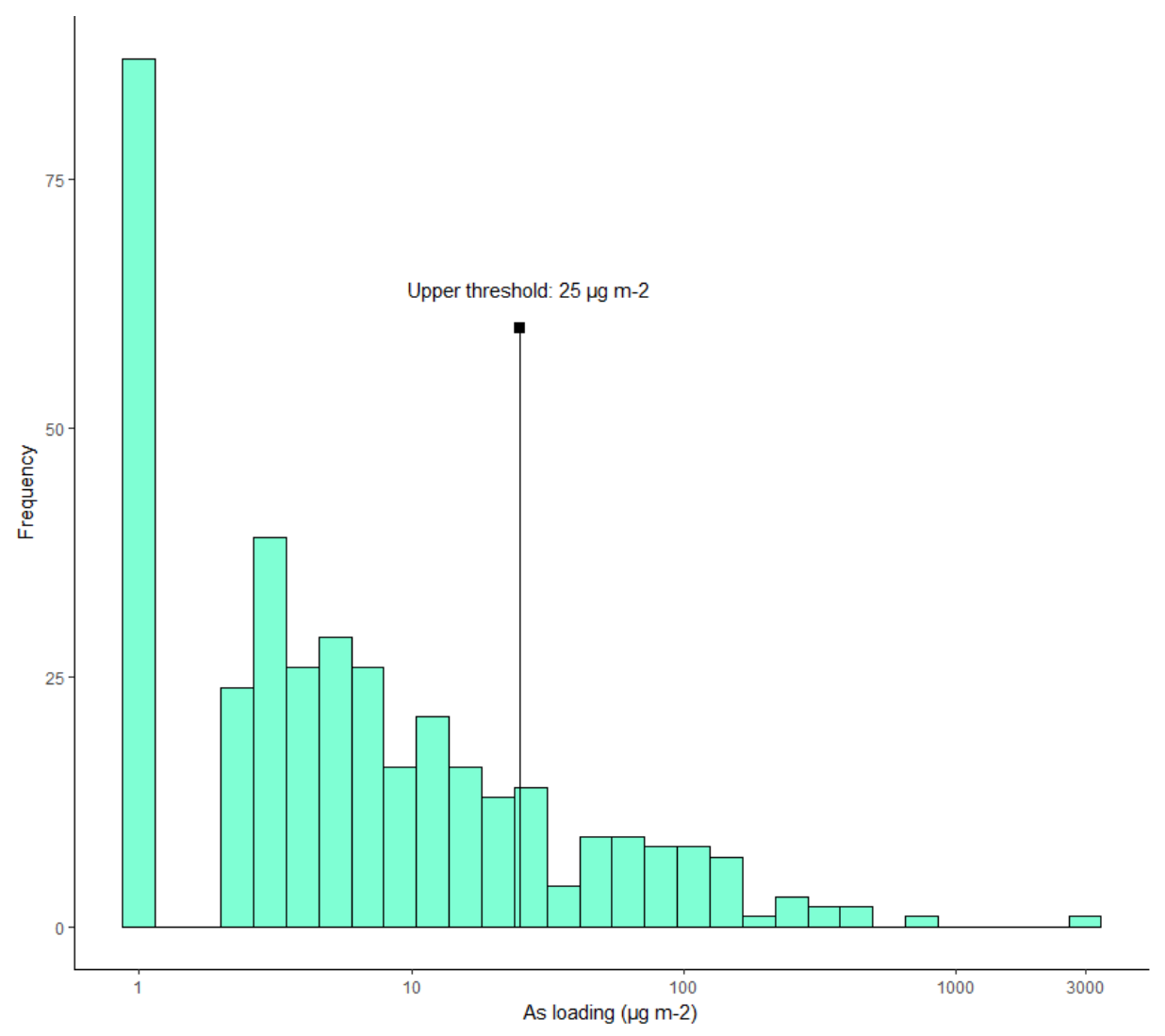

Figure 3 A histogram showing the distribution of As loadings in individual dust wipe samples collected from both study sites. An upper benchmark loading reported by McDonald et al. (2011) is plotted for reference. 


\section{Human exposure assessment}

Figure 4 shows the estimated household daily intake rates of As calculated separately for household mean loadings (wipe sampling) and household bulk concentrations (composite vacuum sampling). In relation to the tolerable daily intake $\left(3.0 \mu \mathrm{g} \mathrm{kg}^{-1} \mathrm{day}^{-1}\right)$, no exceedances were observed using adult dust ingestion rates for either sampling method. Using wipe sampling and infant ingestion rates, one household exceeded the tolerable daily intake. Four (3\%) households exceeded the tolerable daily intake when estimates were made using bulk concentrations and infant ingestion rates.

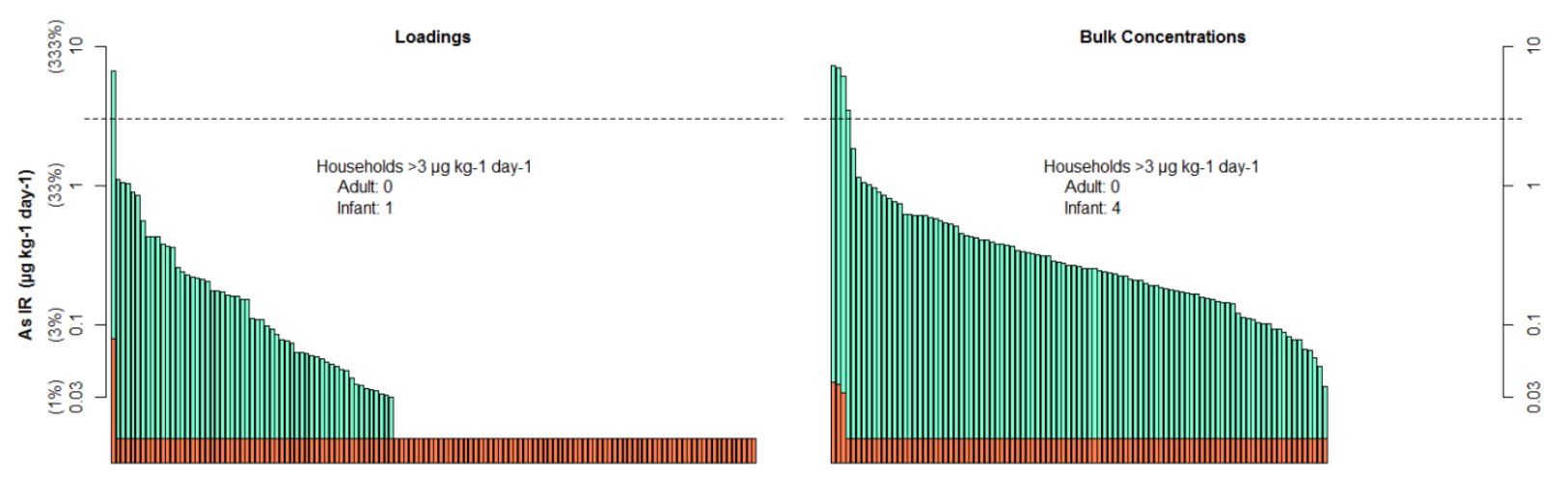

Figure 4 Estimated household daily intake rates of As using household mean wipe loadings and bulk concentrations from composite vacuum samples. Intake rates (for individual households $-\mathrm{x}$ axis) are plotted for adults and infants in relation to the As tolerable daily intake. To aid plot visibility, intake rates equating to $<1 \%$ of the tolerable daily intake were censored to $0.5 \%$ - resulting in bars of equal heights that are visible for adult estimates.

\section{Disparity between wipe and bulk sampling methods}

Estimating daily As intake rates, using either bulk concentrations and ingestion rates or those in units of surface area, yielded different results. A higher portion of household exceedances of the tolerable daily intake was obtained using the bulk approach. Furthermore, the geometric mean (GM) infant intake rate calculated using bulk concentrations was $0.29 \mu \mathrm{g} \mathrm{kg}^{-1}$ day $^{-1}$, compared to a GM of $0.04 \mu \mathrm{g}$ $\mathrm{kg}^{-1} \mathrm{day}^{-1}$ yielded by surface loadings. Correlation analysis was used to assess within-household agreement of dust As levels between sample collection methods. A weak to moderate Spearman correlation was found between mean household loadings and bulk concentrations of As ( $\rho=0.39$; $\mathrm{P}<0.05)$. A conceivable explanation for this disparity is the household locations represented by the different approaches. Vacuum samples contain mostly floor dust compared to the surface dust collected with wipes. Elemental concentrations have been shown to vary by particle size fraction ${ }^{32}$ and between household dust and soil ${ }^{33}$ - of which contributions may differ by sampling location. 
To further assess the relative utility of both dust sampling methods in predicting human exposure, biomonitoring data (available from the same cohort of Cornwall residents) were utilised. Multiple linear regression models were performed using As concentrations in urine (arsenobetaine and osmolality adjusted, 158 participants), hair (75 participants) and toenail (153 participants) samples as the response variable and either bulk As concentrations or mean household As loadings as the predictor variable. Models were adjusted for total As in drinking water, residential soil, age and gender. The adjusted R-squared values from the models were used as the performance metric and are presented in Table 4. Neither dust metric was a significant predictor of urinary As concentration. This is not unexpected given that urinary As concentrations reflect recent (i.e. 2-4 days) exposure ${ }^{34}$ and adult exposure to dust may be too sporadic/infrequent to be reflected in a urinary biomarker. Both bulk concentrations and mean household loadings were a significant predictor of hair and toenail As concentrations, but the adjusted R-squared values obtained from models using bulk concentrations and mean household loadings were too close to distinguish between. When both bulk concentrations and mean household loadings were included as predictor variables for toenail As concentration, both were significant and the adjusted r-squared marginally improved, suggesting that contrasting information may be contributed from each method. This is consistent with the reasoning that different methods reflect exposure from different locations and including both metrics in tandem might yield a more complete exposure assessment.

\section{Limitations of the study}

Dust wipe samples were collected from interior raised surfaces and insufficient sample numbers prevented the comparison of different areas within households. Element loadings have been shown to vary by wipe location. For example, $\mathrm{As}, \mathrm{Pb}, \mathrm{Cd}, \mathrm{Cr}, \mathrm{Cu}, \mathrm{Ni}$ and $\mathrm{Sb}$ loadings were higher in entry locations compared to interior rooms ${ }^{9}$. Wipe locations have also been shown to vary in their relevance to human exposure assessment. In this regard, child blood $\mathrm{Pb}$ concentrations have been found to be more strongly correlated with $\mathrm{Pb}$ loadings in wipes collected from floors than from windowsills ${ }^{7}$. In the present study, daily intake estimates only exceeded tolerable daily limits where calculations were performed for infants - for which exposure is more likely to occur when playing on the floor. It is possible that potential exposure was underestimated by collected wipes from elevated surfaces. Sampling location differences also undermined the comparison between bulk and wipe samples - both likely reflecting dust of different composition.

A further limitation of the wipe sampling methodology was the lack of control over the time allowed for dust to settle on surfaces. Participants were asked to clean surfaces four weeks prior to sampling and record the date. When asked to provide this date, only $40 \%$ of households had complied. Many householders did not know when or if surfaces had been cleaned at all. Trying to control settlement in 
this manner isn't a feasible aim. Seasonal variation and the difference in local climatic factors would likely limit any efforts made to control settlement rates.

The bulk sampling method employed was a crude approach and was assumed to be a composite of household interiors. In reality it likely reflected only floors and, while participants were asked to provide the vacuum cleaner used in the home only, use elsewhere cannot be ruled out, such as in cars or garages. More robust vacuum sampling approaches have been reported in the literature, such as vacuuming from specified dimensions and locations ${ }^{35}$.

The total As concentrations/loadings employed in exposure estimates did not account for bioaccessibility. When As exposure from residential soil was investigated in the same study population, correcting concentrations for bioaccessibility dramatically reduced estimated human exposures ${ }^{17}$. It is likely that exposure was overestimated by the total As values employed in this analysis. Furthermore, the validity of exposure estimates calculated are all subject to the robustness of the input parameters (e.g. ingestion rates developed in a different population in another country). The prevalence and magnitude of human exposure in south west England cannot be quantified by the methods employed, but serve as an indication of the presence of infant exposure in the study region.

The present study population (adults, being private drinking water supply users, from Cornwall), from which biospecimens were collected, is not well suited to assessing the relative utility of alternative dust sampling methods using As biomonitoring data. Overall, exposure to As in dust is lower in this population compared to the contributions of other sources of As exposure, e.g. the GM wipe-based estimate for adult As exposure from dust was $0.0004 \mu \mathrm{g} \mathrm{kg}^{-1}$ day $^{-1}$ compared to $0.03 \mu \mathrm{g} \mathrm{kg}^{-1} \mathrm{day}^{-1}$ from drinking water. Targeting populations in former mining locations such as DGC, where biospecimens were not collected in this study, may be a more suitable approach given the higher observed dust exposures.

\section{Conclusions}

The findings presented suggest that levels of As in household dust in the study region are mostly above detection and guidelines for tolerable daily intake were exceeded in a few households when using infant dust ingestion rates. This matter warrants further investigation and the inclusion of small children in biomonitoring studies. Targeting highly contaminated locations such as former mining sites would be a worthwhile future direction.

Bulk and wipe methodologies yield contrasting results when estimating human elemental exposures. This was probably because different sampling locations were reflected by the two approaches, with bulk samples containing more floor dust and wipes only collected from raised surfaces. Both metrics provided estimates which were predictive and the relative value of each could not be clearly 
distinguished. It can be concluded that whatever method is chosen, careful consideration of sampling locations should be made to capture the most likely sources of human exposure.

Robust laboratory protocols are required to overcome the analytical obstacles associated with wipe sampling. Particular care should be taken to quantify and correct for instrumental interferences (e.g. ICP-MS signal enhancement) resulting from high carbon concentrations in wipe matrices. Background wipe contamination needs reducing, both during manufacturing and sampling/handling. Both wipe and bulk sampling strategies should be designed to target locations that reflect human exposure for the element/contaminant in question (i.e. floors and play areas for infants) and a representative number of samples should be collected.

Wipe sampling is convenient for dust collection and subsequent dissolution, capable of handling large sample throughput, making it a simple and cost effective approach for qualitative human exposure assessment. Further efforts are needed to refine analytical protocols and tailor sampling procedures to specific contaminants. Such advances need to be complemented by robust sampling strategies, tolerable daily intakes for various contaminants and dust ingestion rates.

\section{Disclaimer}

This paper does not reflect the organisational opinions or recommendations of Public Health England (PHE). The methods used in this paper are for research purposes and are not endorsed by PHE for the purpose of human health exposure assessment.

\section{Acknowledgements}

The authors gratefully acknowledge the support of the wider study team throughout the research: Louise Ander, Amy Rimell, Karen Exley, Mike Studden, Chiara Petrillo, Andrew Marriott, Andrew Dunne, Simon Chenery, Thomas Barlow and Amanda Gardner. Research participants are thanked for their time and contribution. The advice of Pat Rasmussen and Owen Butler was highly valuable to the study design. Funding for this research was provided by the Natural Environment Research Council (NERC) via a University of Manchester/British Geological Survey (BGS) University Funding Initiative (BUFI) PhD studentship (Contract No. GA/125/017, BUFI Ref: S204.2) and the Centre for Environmental Geochemistry, BGS. 


\section{References}

1. WHO, 2010. Exposure To Arsenic: A Major Public Heath Concern. Public Health and Environment, World Health Organization. Geneva, Switzerland. Available: http://www.who.int/ipcs/features/arsenic.pdf

2. A. Navas-Acien, E. K. Silbergeld, R. Pastor-Barriuso and E. Guallar, Jama, 2008, 300, 814822.

3. A. Navas-Acien, A. R. Sharrett, E. K. Silbergeld, B. S. Schwartz, K. E. Nachman, T. A. Burke and E. Guallar, American journal of epidemiology, 2005, 162, 1037-1049.

4. $\quad$ C.-J. Chen, Y.-M. Hsueh, M.-S. Lai, M.-P. Shyu, S.-Y. Chen, M.-M. Wu, T.-L. Kuo and T.Y. Tai, Hypertension, 1995, 25, 53-60.

5. IARC., IARC Monogr Eval Carcinog Risks Hum., 2012, 100C, 41-85.

6. ATSDR, 2015. Agency for Toxic Substances and Disease Registry (ATSDR) Substance Priority List. Available: http://www.atsdr.cdc.gov/spl/

7. J. Wilson, S. Dixon, W. Galke and P. McLaine, J Expos Sci Environ Epidemiol, 2006, 17, 212.

8. M. Maddaloni, N. Jeffery, P. Grevatt, K. Carlino, T. Smith, J. Prudhomme, D. LaPosta, C. D'Andrea, J. Girman and V. Colucci, 2003. World Trade Center Indoor Air Assessment: Selecting Contaminants of Potential Concern and Setting Health-Based Benchmarks.

9. L. T. McDonald, P. E. Rasmussen, M. Chénier and C. Levesque, Journal of Environmental Monitoring, 2011, 13, 377-383.

10. M. P. Taylor, D. Camenzuli, L. J. Kristensen, M. Forbes and S. Zahran, Environmental pollution, 2013, 178, 447-454.

11. F. Barrio-Parra, E. De Miguel, S. Lázaro-Navas, A. Gómez and M. Izquierdo, Exposure and Health, 2017, DOI: 10.1007/s12403-017-0244-z, 1-10.

12. P. E. Rasmussen, C. Levesque, M. Chénier, H. D. Gardner, H. Jones-Otazo and S. Petrovic, Science of The Total Environment, 2013, 443, 520-529.

13. R. Wilson, H. Jones-Otazo, S. Petrovic, I. Mitchell, Y. Bonvalot, D. Williams and G. M. Richardson, Human and Ecological Risk Assessment: An International Journal, 2013, 19, 158-188. 
14. R. Wilson, I. Mitchell and G. M. Richardson, Human and Ecological Risk Assessment: An International Journal, 2016, 22, 874-881.

15. H. G. Dines, The metalliferous mining region of south-west England, HM Stationery Office, 1956.

16. P. Abrahams and I. Thornton, Institution of Mining and Metallurgy Transactions. Section B. Applied Earth Science, 1987, 96.

17. D. R. Middleton, M. J. Watts, D. J. Beriro, E. M. Hamilton, G. S. Leonardi, T. Fletcher, R. M. Close and D. A. Polya, Environmental Science: Processes \& Impacts, 2017. DOI: 10.1039/C6EM00690F

18. E. Ander, M. Watts, P. Smedley, E. Hamilton, R. Close, H. Crabbe, T. Fletcher, A. Rimell, M. Studden and G. Leonardi, Environmental geochemistry and health, 2016, 1-20.

19. D. Middleton, M. Watts, E. Hamilton, E. Ander, R. Close, K. Exley, H. Crabbe, G. Leonardi, T. Fletcher and D. Polya, Scientific Reports, 2016. DOI:10.1038/srep25656

20. D. Middleton, M. Watts, E. Hamilton, T. Fletcher, G. Leonardi, R. Close, K. Exley, H. Crabbe and D. Polya, Environmental Science: Processes \& Impacts, 2016. DOI: 10.1039/c6em00072j

21. M. Button, G. R. Jenkin, C. F. Harrington and M. J. Watts, Journal of Environmental Monitoring, 2009, 11, 610-617.

22. D. F. Peach and D. W. Lane, Environmental Geochemistry and Health, 1998, 20, 231-237.

23. J. S. Rieuwerts, P. Searle and R. Buck, Science of The Total Environment, 2006, 371, 89-98.

24. British Geological Survey, Arsenic: are we exposed? http://www.bgs.ac.uk/sciencefacilities/laboratories/geochemistry/igf/Biomonitoring/arsenicSW.html, accessed: 25/09/2017).

25. H. Crabbe, Close, R., Rimmell, A., Leonardi, G., Watts, M.J., Ander, E.L., Hamilton, E.M., Middleton, D.R.S., Smedley, P.L., Gregory, M., Robjohns, S., Sepai, O., Studden, M., Polya, D.A., and Fletcher, T., 2016. in Bhattacharya, P. Polya, D.A. and Jovanovic. D. (Eds.) Best Practice Guide for the Control of Arsenic in Drinking Water, IWA Publishing, Chapter A4, ISBN13: 9781843393856 .

26. ASTM, 2002. ASTM (American Society for Testing and Materials), Report No. E 1728-02, Standard Practice for Collection of Settled Dust Samples Using Wipe Sampling Methods for Subsequent Lead Determination, West Conshohocken, Pennsylvania, United States, 2002. 
27. E. M. Hamilton, T. S. Barlow, C. J. Gowing and M. J. Watts, Microchemical Journal, 2015, 123, 131-138.

28. RCoreTeam, 2016. R: A language and environment for statistical computing. R Foundation for Statistical Computing, Vienna, Austria. URL https://www.R-project.org/.

29. RStudioTeam, 2015. RStudio: Integrated Development for R. RStudio, Inc., Boston, MA URL http://www.rstudio.com/.

30. J. Jeffries and I. Martin, Updated technical background to the CLEA model, Environment Agency, 2009.

31. J. J. Keenan, M. H. Le, D. J. Paustenbach and S. H. Gaffney, Bulletin of environmental contamination and toxicology, 2010, 84, 269-273.

32. C. Lanzerstorfer, Journal of Environmental Science and Health, Part A, 2017, 1-8.

33. P. I. Beamer, C. A. Elish, D. J. Roe, M. M. Loh and D. W. Layton, Journal of Environmental Monitoring, 2012, 14, 839-844.

34. K. Orloff, K. Mistry and S. Metcalf, Journal of Toxicology and Environmental Health, Part B, $2009,12,509-524$.

35. P. E. Rasmussen, K. S. Subramanian and B. J. Jessiman, Science of The Total Environment, 2001, 267, 125-140. 
Table 1 Revised mean daily dust ingestion rates for adults and infants calculated by Wilson et al. $(2013,2016)$ in units of surface area and bulk concentration. Estimates assume $50 \%$ hard and $50 \%$ soft household surfaces.

\begin{tabular}{|l|c|c|}
\hline Age group & Dust ingestion rate $\left(\mathrm{m}^{2} \mathrm{day}^{-1}\right)$ & Dust ingestion rate $\left(\mathrm{mg} \mathrm{day}^{-1}\right)$ (converted to $\mathrm{mg} \mathrm{day}^{-1}$ prior to calculations) \\
\hline $\begin{array}{l}\text { Adult } \\
\text { (20-59 years) }\end{array}$ & 0.0037 & 2.5 \\
\hline $\begin{array}{l}\text { Infant } \\
\text { (0-6 } \text { months) }\end{array}$ & 0.025 & 38 \\
\hline
\end{tabular}

Table 2 Mean As recoveries and precision (percentage relative standard deviation) of CRM-spiked dust wipes and a matrix-matched QC standards analysed throughout the study.

\begin{tabular}{|l|c|c|}
\hline \multicolumn{1}{|c|}{ CRM } & Replicates & Mean percentage recovery (\% RSD) \\
\hline $\begin{array}{l}\text { NIST 2584 } \\
\text { Indoor Dust }\end{array}$ & 40 & $86(13)$ \\
\hline $\begin{array}{l}\text { NIST 2711a } \\
\text { Montana II } \\
\text { Soil }\end{array}$ & 40 & $107(8)$ \\
\hline $\begin{array}{l}\text { Matrix- } \\
\text { matched } \\
\text { QC } \\
\left(25 \mu \mathrm{L} \mathrm{L}^{-1}\right)\end{array}$ & 27 & $81(3)$ \\
\hline
\end{tabular}


Table 3 Summary statistics for As loadings determined in individual wipe samples and the proportion of wipes and households exceeding the limit of detection (LOD), an upper benchmark loading reported by McDonald et al. (2011).

\begin{tabular}{|l|c|c|}
\hline Study site & Geometric mean (range) loading $\left(\mu \mathrm{g} \mathrm{m}^{-2}\right)$ & $\begin{array}{c}n \text { wipes >upper benchmark } \\
(\boldsymbol{n} \text { households; \%) }\end{array}$ \\
\hline $\begin{array}{l}\text { Cornwall- } \\
\text { wide }\end{array}$ & 5.6 & 58 \\
\hline DGC & $(<$ LOD-2,980) & $(33 ; 26 \%)$ \\
\hline
\end{tabular}

Table 4 Goodness of fit of multiple regression models using either bulk concentrations or surface As loadings as predictor variables of urine, hair and toenail As concentrations. Estimated coefficients are shown in brackets with their significance denoted as follows: ${ }^{* * *} P<0.001 ;{ }^{* *} P<0.01 ;{ }^{*} P<0.05$. All environmental and biological As variables were natural log transformed. All models were adjusted for age and gender.

\begin{tabular}{|c|c|c|}
\hline Biomarker response variable & Environmental predictor variables & Adjusted R-squared \\
\hline Urinary As & Bulk dust $\mathrm{As}(0.07)+$ Drinking water $\mathrm{As}\left(0.22^{* * *}\right)+$ Soil $\mathrm{As}(0.04)$ & 0.42 \\
\hline Urinary As & Mean As loading $(0.03)+$ Drinking water $\mathrm{As}(0.23 * * *)+$ Soil $\mathrm{As}(0.06)$ & 0.42 \\
\hline Hair As & Bulk dust $\mathrm{As}(0.30 *)+$ Drinking water $\mathrm{As}(0.20 * *)+$ Soil $\mathrm{As}(-0.09)$ & 0.41 \\
\hline Hair As & Mean As loading $\left(0.20^{* *}\right)+$ Drinking water $\operatorname{As}(0.22 * *)+$ Soil $\operatorname{As}(0.01)$ & 0.43 \\
\hline Toenail As & Bulk dust $\mathrm{As}(0.31 * * *)+$ Drinking water $\mathrm{As}(0.19 * * *)+\operatorname{Soil} \mathrm{As}(0.22 * * *)$ & 0.51 \\
\hline Toenail As & Mean As loading $\left(0.20^{* * *}\right)+$ Drinking water As $\left(0.21^{* * *}\right)+$ Soil As $(0.29 * * *)$ & 0.49 \\
\hline Toenail As & Mean As loading $\left(0.12^{* *}\right)+$ Bulk dust As $(0.22 * * *)+$ Drinking water $\mathrm{As}(0.19 * * *)+$ Soil $\mathrm{As}(0.24 * * *)$ & 0.54 \\
\hline
\end{tabular}

